\title{
The Impact of G.826 on the Performance of Transport Systems
}

\author{
Peter J Smith, Member, IEEE, and Mansoor Shafi, Fellow, IEEE
}

\begin{abstract}
Study Group XIII of the ITU-TS has recently approved a new performance recommendation G.826 which the ITU-R has recommended as a standard for satellite systems in recommendation S.1062. G.826 establishes guidelines on the performance levels of digital systems operating at or above primary rate. These performance levels are stated in terms of block error rates. In this paper, we discuss the impact of the block-based error performance objectives on the performance of transport systems. However, the impact of G.826 is difficult to evaluate directly since the performance recommendations are not given in terms of bit-error rate (BER) values. Hence, we present a procedure to convert block error rates to BER. This conversion is based on the statistical modeling of the error process and includes an analysis of bursty errors as well as random errors. Rather than using the equivalent BER values as simple thresholds, we produce BER against time distributions which satisfy G.826 and can be used as masks for a comparison with measured data and also for route design. It is shown that these masks are a crucial tool in any study of G.826 and contain useful information for system design and planning. Indeed it is shown that false conclusions can be drawn if the masks are not used. Finally, the impact of G.826 is discussed with particular reference to apportionment strategies, system margins, signal processing, and error correction schemes.
\end{abstract}

\section{INTRODUCTION}

$\mathbf{S}^{\mathrm{T}}$ TUDY GROUP XIII of the ITU-TS has recently approved new performance recommendation on the subject of error performance for digital systems operating at or above the primary rate [1]. The new performance recommendation is a significant departure from its precursor, G.821 [2], in the sense that objectives are media-independent, block-based, and suitable for doing in-service measurements (ISM's). In addition, the apportionment of the performance objectives to the various parts of the network is left up to the operators. The motivation for the preparation of G.826 is the move toward providing broadband services to customers. Based on the work of the ITU-T sector, the ITU-R sector is developing recommendations for the performance of radio relay and satellite links which will meet G.826. For example, Recommendation S.1062 [3] states that satellite systems within the public switched networks operating at or above the primary rate should be designed to meet ITU-T Recommendation G.826.

To evaluate the impact of G.826, it is essential to convert the block-based objectives to BER criteria. This is necessary

Manuscript received June 9, 1995; revised April 3, 1996; approved by IEEE/ACM TRANSACTIONS ON NETwORKING Editor P. O'Reilly.

P. J. Smith is with the Institute of Statistics and Operations Research, Victoria University of Wellington, Wellington, New Zealand.

M. Shafi is with the Fundamental Planning, Telecom New Zealand Ltd, Telecom House, Manners Street, Wellington, New Zealand.

Publisher Item Identifier S 1063-6692(96)06082-7. for comparing the stringency of G.821 and G.826 error performance objectives (EPO's), checking to see if equipment specifications (which often quote receiver sensitivities in terms of BER) will comply with G.826, and assessing network performance as per G.826 from BER data and distributions. Previous work in this area has only given results based on a constant BER assumption [4]. This is useful as an initial study of G.826 but is far from a complete analysis. In this paper, we present and implement two essential tools for a complete assessment of G.826. These are:

- a mathematical derivation of the relationship between block-based error performance parameters and the BER;

- a simple algorithm to compute BER distributions over time (masks) which satisfy G.826. ${ }^{1}$

Using these tools we can gain a new and more accurate picture of the impact of G.826. In this paper, we specifically address the following questions.

- In the design of transmissions systems it is sufficient to assume a constant BER or is it necessary to consider BER distributions over time?

- How should the error performance objectives be apportioned between the customer access network (CAN) and the domestic interexchange network (IEN)?

- What are the BER requirements resulting from G.826 for the various sections of an international digital path?

- What are the impacts of these BER requirements on various transport media?

The format of this paper is as follows. In Section II, the main points of G.826 are discussed. The conversion of the block-based error performance objectives to BER criteria is derived in Section III. In Section IV, we produce masks for the resulting BER distributions as well as simple BER thresholds and discuss the implications of G.826. In addition, various scenarios are considered for the apportionment of the performance objectives to the different parts of the network. Finally, in Section V some conclusions are presented.

\section{RECOMMENDATION G.826}

The development of G.826 from its precursor, G.821, is discussed in more detail in [4]. In this section we simply outline the main points of G.826. The error performance events and parameters in G.826 are the following.

- Errored Block (EB): a block in which one or more bits are in error.

${ }^{1}$ Note that Rec. S.1062 specifically considers BER design masks so this methodology is of increasing importance and is discussed in Section IV. 


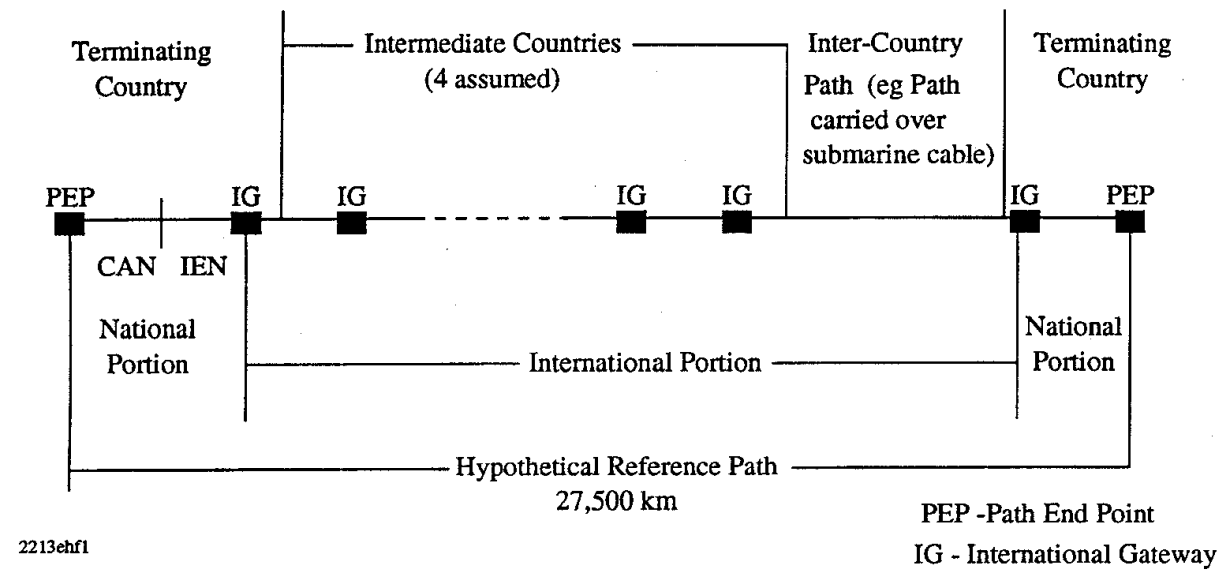

Fig. 1. Hypothetical reference path.

TABLE I

Error Performance ObJectives for G.826

\begin{tabular}{ccccc}
\hline Rate $(\mathrm{Mb} / \mathrm{s})$ & Bits/block & ESR & SESR & BBER \\
\hline $1.5-5$ & $2000-8000$ & 0.04 & 0.002 & $3 \times 10^{-4}$ \\
$>5-15$ & $2000-8000$ & 0.05 & 0.002 & $2 \times 10^{-4}$ \\
$>15-55$ & $4000-20000$ & 0.075 & 0.002 & $2 \times 10^{-4}$ \\
$>55-160$ & $6000-20000$ & 0.16 & 0.002 & $2 \times 10^{-4}$ \\
$>160-3500$ & $15000-30000$ & $* *$ & 0.002 & $10^{-4}$ \\
$>3500$ & FFS $^{*}$ & FFS $^{*}$ & FFS* & FFS* \\
\hline
\end{tabular}

${ }^{*}$ FFS: for further study

${ }^{* *}$ No objective given due to the lack of available information

- Errored Second (ES): a one second period with one or more errored blocks.

- Severely Errored Second (SES): a one second period that contains $\geq 30 \%$ EB's or at least one severely disturbed period (SDP). For out-of-service measurements, an SDP occurs when, over a minimum period of time equivalent to four contiguous blocks, either all the contiguous blocks are affected by a high binary error density of $10^{-2}$, or a loss of signal information is observed. For inservice monitoring purposes, an SDP is estimated by the occurrence of a network defect. The term "defect" is defined in the relevant annexes (2, 3, or 4/G.826) for the different network fabrics-PDH, SDH, or cell-based, respectively.

- Background Block Error (BBE): an EB not occurring as part of an SES.

- ES Ratio (ESR): the ratio of ES's to total seconds in available time during a fixed measurement interval.

- SES Ratio (SESR): the ratio of SES's to total seconds in available time during a fixed measurement interval.

- BBE Ratio (BBER): The ratio of EB's to total blocks during a fixed measurement interval, excluding all blocks during SES's and unavailable time.

1) EPO's: The EPO's proposed in G.826 are summarized in Table I. All objectives are measured over "available" time in a fixed measurement interval (one month recommended). All three objectives (ESR, SESR, and BBER) must hold concurrently to satisfy G.826, and apply end-to-end for a $27500 \mathrm{~km}$ hypothetical reference path (HRP).
TABLE II

APPORTIONMENT RULES FOR G.286

\begin{tabular}{|c|c|c|c|}
\hline \multicolumn{4}{|c|}{$\%$ of EPO } \\
\hline \multicolumn{2}{|c|}{ National portion* } & \multicolumn{2}{|c|}{ International portion ${ }^{*}$} \\
\hline Block allowance & $\begin{array}{l}\text { Distance } \\
\text { allowance }\end{array}$ & Transit allowance** & $\begin{array}{l}\text { Distance } \\
\text { allowance }\end{array}$ \\
\hline $\begin{array}{l}17.5 \% \text { to both } \\
\text { terminating } \\
\text { countries }\end{array}$ & $1 \%$ per $500 \mathrm{~km}$ & $\begin{array}{l}2 \% \text { per intermediate } \\
\text { country } \\
1 \% \text { per terminating } \\
\text { country }\end{array}$ & $\begin{array}{l}1 \% \text { per } 500 \\
\mathrm{~km}\end{array}$ \\
\hline
\end{tabular}

* Satellite hops each receive $35 \%$ but the distance of the hop is removed from the distance allowance.

${ }^{* *}$ Four intermediate countries are assumed.

2) Availability: The concept of available time is defined below. Unavailable time commences at the start of a block of ten consecutive SES's. The unavailable time finishes (and available time begins) at the start of a block of ten consecutive seconds, each of which is not severely errored.

3) Apportionment: Table II gives details of the apportionment rules. Fig. 1 shows a schematic HRP with the different sections of the HRP discussed in the apportionment rules.

\section{THE RELATIONSHIP BETWEeN G.826 EPOS AND EQUIVALENT BER}

It is useful to express the G.826 EPO's in terms of BER values. The relationship between G.826 EPO's and BER is dependent on the choice of models to describe the occurrence of errors. The errors can occur randomly or in bursts. Systems using forward error correction (e.g., satellite systems and digital radio systems) and/or adaptive equalization (e.g., digital radio systems) are particularly susceptible to burst errors. The derivation of relationships between the EPO's and BER is quite complex for both error occurrence mechanisms. The following notation is needed before we begin the analysis.

\section{A. Notation}

$M \quad$ Number of blocks per second.

$R \quad$ Number of bits per second.

$\Delta t \quad 1 / M$

$p \quad$ BER 


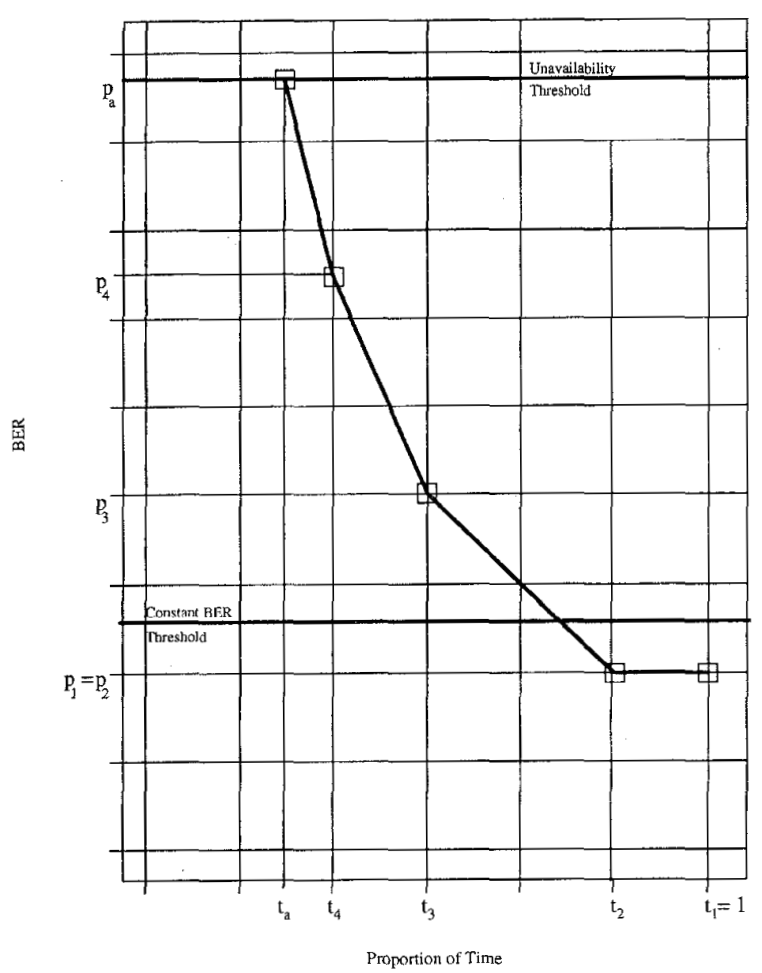

Fig. 2. Schematic mask.

EFS Error free second.

SEB Severely errored block (a block having an error density $\geq 10^{-2}$ ).

NSEB Nonseverely error block (a block which is an EB but not an SEB).

$E_{1} \quad$ Event that $\geq 30 \%$ of the blocks in one second are errored.

$E_{2} \quad$ Event that an SDP occurs in the second.

$N \quad$ Smallest integer $\geq 30 \%$ of $M$.

\section{B. Random Errors}

Here, we assume bits are independent with constant probability, $p$, of error.

ESR:

$$
\mathrm{ESR}=P(\mathrm{ES})=1-P(\mathrm{EFS})=1-(1-p)^{R} .
$$

BBER:

$$
\begin{aligned}
\mathrm{BBER} & =P(\mathrm{BBE}) \\
& =P(\mathrm{~EB} \text { not occurring in an SES or } \\
& \quad \text { unavailable time }) \\
& \approx P(\mathrm{~EB} \text { and the second is not an SES })
\end{aligned}
$$

neglecting the unavailability condition which has a negligible effect on the probability.

Hence

$$
\begin{aligned}
\mathrm{BBER}= & P\left(\mathrm{~EB} \cap \bar{E}_{1} \cap \bar{E}_{2}\right) \\
= & \sum_{i=0}^{N} \sum_{j=0}^{i} \sum_{k=r}^{j} \frac{i}{M} N(j, k)\left(\begin{array}{c}
M-j+1 \\
k
\end{array}\right)\left(\begin{array}{c}
M-j \\
i-j
\end{array}\right) \\
& \cdot p_{\mathrm{SEB}}^{j} p_{\mathrm{NSEB}}^{i-j}\left(1-p_{\mathrm{EB}}\right)^{M-i}
\end{aligned}
$$

where $N(j, k)$ is the number of ways in which the $j$ SEB's can be put in $k$ nonconsecutive groups such that the maximum number of SEB's in a group is three. The smallest possible number of groups is $r$, where $r=j / 3$ if $j$ is a multiple of three and $r$ is the smallest integer $\geq j / 3$ otherwise. It is fairly simple to derive the following recurrence formula for the $N(j, k)$ terms

$$
\begin{aligned}
N(j, k) & =\sum_{i=i_{1}}^{i_{2}} N(j-i, k-1) \\
i_{1} & =\max (1, j-3 k+3) \\
i_{2} & =\min (3, j-k+1) \\
N(0,0) & =1=N(1,1)=N(2,1)=N(3,1) .
\end{aligned}
$$

Full details of the derivation of (2) are given in Appendix A. The three probabilities in (2) are easily computed and are given by

$$
\begin{aligned}
p_{\mathrm{EB}} & =1-(1-p)^{R \Delta t} \\
p_{\mathrm{SEB}} & =\sum_{i=L}^{R \Delta t}\left(\begin{array}{c}
R \Delta t \\
i
\end{array}\right) p^{i}(1-p)^{R \Delta t-i} \\
& \quad \text { where } L \text { is the smallest integer } \geq \frac{R \Delta t}{100} \\
p_{\mathrm{NSEB}}= & p_{\mathrm{EB}}-p_{\mathrm{SEB}} .
\end{aligned}
$$

The triple summation involved in computing the BBER can be numerically troublesome so that the following approximation is useful.

$$
\begin{aligned}
\mathrm{BBER} & \leq P\left(\mathrm{~EB} \cap \bar{E}_{1}\right) \\
& =\sum_{i=0}^{N-1} P(\mathrm{~EB} \text { and } i \text { EBs in } 1 \text { second }) \\
& =\sum_{i=0}^{N-1} \frac{i}{M}\left(\begin{array}{c}
M \\
i
\end{array}\right) p_{\mathrm{EB}}^{i}\left(1-p_{\mathrm{EB}}\right)^{M-i}
\end{aligned}
$$

where the bar notation in $\bar{E}_{1}$ denotes the complement of $E_{1}$

$$
\begin{aligned}
\mathrm{BBER} & =1-P(\overline{\mathrm{EB}} \cup \mathrm{SES}) \\
& \geq 1-P(\overline{\mathrm{EB}})-p_{\mathrm{SES}}=p_{\mathrm{EB}}-p_{\mathrm{SES}} .
\end{aligned}
$$

From (3) and (4), tight bounds on the BBER can be computed which are significantly easier to produce than equation (2).

SESR:

$$
\begin{aligned}
\mathrm{SESR}= & P(\mathrm{SES}) \\
= & P\left(E_{1} \cup E_{2}\right) \\
& \text { neglecting the unavailability condition } \\
= & 1-P\left(\bar{E}_{1} \cap \bar{E}_{2}\right) \\
= & 1-\sum_{i=0}^{N-1} \sum_{j=0}^{i} \sum_{k=r}^{j} N(j, k)\left(\begin{array}{c}
M-j+1 \\
k
\end{array}\right)\left(\begin{array}{c}
M-j \\
i-j
\end{array}\right) \\
& \cdot p_{\mathrm{SEB}}^{j}\left(1-p_{\mathrm{EB}}\right)^{M-i} p_{\mathrm{NSEB}}^{i-j}
\end{aligned}
$$

where $r$ and $N(j, k)$ are defined as for the BBER. The derivation of (5) is found in Appendix B. Again the triple 
summation can cause problems so that an approximation is useful

$$
P\left(E_{1}\right) \leq \mathrm{SESR} \leq \min \left(1, P\left(E_{1}\right)+P\left(E_{2}\right)\right)
$$

where

$$
P\left(E_{1}\right)=1-\sum_{i=0}^{N-1}\left(\begin{array}{c}
M \\
i
\end{array}\right) p_{\mathrm{EB}}^{i}\left(1-p_{\mathrm{EB}}\right)^{M-i}
$$

and $P\left(E_{2}\right)$ can be derived using standard results on runs probabilities [5]

$$
P\left(E_{2}\right)=1-u_{M}
$$

where $u_{0}=u_{1}=u_{2}=u_{3}=1, u_{4}=1-p_{\mathrm{SEB}}^{4}$ and $u_{j}=u_{j-1}-\left(1-p_{\mathrm{SEB}}\right) p_{\mathrm{SEB}}^{4} u_{j-5}$ for $j \geq 5$.

In [4], it is shown that $P\left(E_{2}\right)$ is negligible compared to $P\left(E_{1}\right)$ and hence $P\left(E_{1}\right)$ can also be used as an approximation for SESR.

\section{Burst Errors}

There are many ways to model the occurrence of bursts of errors in digital transmissions. For low BER values it is interesting that most approaches yield very similar results. However, in the calculation of masks (see Section IV) it is important to compute the EPO accurately even for high BER values around the unavailability threshold. In these situations the more complex models may be preferable. We present three models below, each of which is useful in different situations. In each case, the mean number of errors in each burst is $\mu$.

1) Model 1: This is the simplest possible model where each bit has constant probability $p_{b}=p / \mu$ of being the first errored bit in a burst of $\mu$ consecutive errors. Hence, the model overcounts as bursts can overlap. Also blocks are considered independently so that we do not consider the possibility of bursts affecting more than one block. With these primitive assumptions the results follow directly from the formulae for random errors. If $p_{\mathrm{SEB}}$ is redefined as

$$
\begin{aligned}
p_{\mathrm{SEB}}= & \sum_{i=K}^{R \Delta t}\left(\begin{array}{c}
R \Delta t \\
i
\end{array}\right) p_{b}^{i}\left(1-p_{b}\right)^{R \Delta t-i} \\
& \text { where } K \text { is the smallest integer } \geq \frac{R \Delta t}{100 \mu}
\end{aligned}
$$

then ESR, BBER, and SESR are still given by (1), (2)-(4), and (5)-(8), respectively, if $p$ is replaced by $p_{b}$.
2) Model 2: This is a well known model discussed by Pullum [6] where the occurrence and length of bursts are modeled by independent Poisson distributions. The resulting compound Poisson distribution is often described as Neyman's Type A distribution. This model also overcounts since error bursts can overlap.

As in Model 1, each bit has a constant probability $p_{b}$ of starting a burst of errors. In this paper we say the length of each burst is $Y+1$ where $Y$ has a Poisson distribution with parameter $\mu-1$. This slight alteration to Neyman's distribution is to avoid the occurrence of bursts of length zero. The burst error probability, $p_{b}$, can be related to the BER by the approximation $p_{b}=p / \mu$ or by the exact result derived below which adjusts for overcounting.

A bit is error free if it does not start a burst of errors and no previous bits start a burst of errors which include it. Since the $j$ th previous bit has probability $p_{b} P(Y \geq j)$ of starting a burst which covers the bit in question we can write

$$
p=1-\prod_{j=0}^{\infty}\left[1-p_{b} P(Y \geq j)\right] \text {. }
$$

ESR:

$$
\operatorname{ESR}=1-\left(1-p_{b}\right)^{R}
$$

SESR: It is possible to analyze the $E_{1}$ event with great accuracy so that the bound SESR $\geq P\left(E_{1}\right)$ in (6) is convenient to compute. Various approximations discussed later under Model 3 show that as in the random error case $P\left(E_{2}\right)$ is negligible compared to $P\left(E_{1}\right)$ and so this bound is usually sufficient.

We set up a three state Markov chain defined on the blocks in a one second period. The dependence modeled by the Markov chain allows bursts of errors to affect consecutive blocks. Details are given in the table at the bottom of the page.

With these state definitions the transition matrix can be written

$$
\left[\begin{array}{ccc}
1-p_{\mathrm{EB}} & 0 & p_{\mathrm{EB}} \\
1-p_{\mathrm{EB}} & 0 & p_{\mathrm{EB}} \\
q & r & p_{\mathrm{EB}}
\end{array}\right]
$$

where $p_{\mathrm{EB}}$ is given by

$$
p_{\mathrm{EB}}=1-\left(1-p_{b}\right)^{R \Delta t}
$$

and $r$ is derived below

$$
\begin{gathered}
r=P(\mathrm{~EB} \text { and no bursts begin in the block } \mid \geq 1 \\
\text { bursts begin in the previous block }) \\
=\frac{1-p_{\mathrm{EB}}}{p_{\mathrm{EB}}} P(\geq 1 \text { bursts in the previous block } \\
\text { overlap the current block. })
\end{gathered}
$$

\begin{tabular}{|c|l|}
\hline State of Block & Interpretation \\
\hline 0 & block is error free \\
1 & block is errored and no bursts of errors begin in the block \\
2 & block is errored and $\geq \mathbf{1}$ bursts begin in the block \\
\hline
\end{tabular}


Now define

$\pi_{j}=P(\geq 1$ bursts in the first $j$ bits of

the previous block overlap the current block.)

The $\pi_{j}$ can be computed recursively from

$$
\begin{aligned}
& \pi_{j}=\pi_{j-1}+\left(1-\pi_{j-1}\right) p_{b} P(Y \geq R \Delta t-j+1) \\
& \pi_{0}=0
\end{aligned}
$$

and the formula for $r$ becomes

$$
r=\frac{1-p_{\mathrm{EB}}}{p_{\mathrm{EB}}} \pi_{R \Delta t} .
$$

With the Markov chain so defined we can write

$$
\begin{aligned}
P\left(E_{1}\right) & =P(\text { state } 0 \text { is visited } \leq M-N \text { times in } M \text { steps }) \\
& =\sum_{k=0}^{M-N} P\left(N_{00}^{(M)}=k\right)
\end{aligned}
$$

where $N_{00}^{(M)}$ is the number of visits to state zero, starting from state zero, in $M$ steps. The computation of occupancy probabilities such as those in (13) is well known and an excellent discussion is given by Hunter [7]. Following his approach, based on certain generating functions gives

$$
\begin{aligned}
P\left(N_{00}^{(M)}=0\right)= & \frac{\beta^{M+1}-\alpha^{M+1}}{\beta-\alpha} \\
P\left(N_{00}^{(M)}=k\right)= & \left(1-p_{\mathrm{EB}}\right)^{k} \sum_{i=0}^{\min (M-k, k)} \sum_{j=0}^{M-k-i}\left(\begin{array}{c}
k \\
i
\end{array}\right) \\
& \cdot\left(\begin{array}{c}
k+j \\
j
\end{array}\right)\left(\begin{array}{c}
M-i-j \\
M-k-i-j
\end{array}\right)(-\gamma)^{i} \alpha^{j} \\
& \cdot \beta^{M-k-i-j}= \\
\alpha= & \frac{1}{2}\left(p_{\mathrm{EB}}-\sqrt{p_{\mathrm{EB}}^{2}+4 r p_{\mathrm{EB}}}\right) \\
\beta= & \frac{1}{2}\left(p_{\mathrm{EB}}+\sqrt{p_{\mathrm{EB}}^{2}+4 r p_{\mathrm{EB}}}\right) \\
\gamma= & \frac{r p_{\mathrm{EB}}}{1-p_{\mathrm{EB}}} .
\end{aligned}
$$

Implementation of (13) requires a triple summation which can be numerically intensive. A normal approximation to (13) can be used as discussed in [7]. This is given by

$$
P\left(E_{1}\right) \approx Q\left(\frac{M\left(1-p_{\mathrm{EB}}-r p_{\mathrm{EB}}\right)-M+N-\frac{1}{2}}{\sqrt{M p_{\mathrm{EB}}(1+3 r)\left(1-p_{\mathrm{EB}}-r p_{\mathrm{EB}}\right)}}\right)
$$

where $Q(\cdot)$ is the standard Gaussian tail probability function.

BBER: If we consider blocks independently, then the BBER is approximated by (2) where $p_{\mathrm{EB}}$ and $p_{\mathrm{SEB}}$ are given by (12) and (9) respectively. However, the bounds given by (3) and (4) are more convenient and can be used directly here by using (12) for $p_{\mathrm{EB}}$ and (13) or (14) for $p_{\mathrm{SES}}$.

3) Model 3: Models 1 and 2 can be used to give perfectly adequate results for the ESR and BBER EPO's. For the SESR, however, we have used the approximation SESR $\approx P\left(E_{1}\right)$. To verify this, we must be able to compute $P\left(E_{2}\right)$ in order to show it is negligible compared to $P\left(E_{1}\right)$. Neglecting the effect of bursts affecting more than one block then (8) can be used for $P\left(E_{2}\right)$ if $p_{\mathrm{SEB}}$ is redefined. To compute $p_{\mathrm{SEB}}$ for bursts of errors neither Models 1 or 2 are completely satisfactory. It is more accurate to model the length of bursts as a Poisson variable and the distance between bursts as a geometric variable. This is Model 3 and it has the advantage of avoiding any overcounting problems. Results from this model demonstrate that $P\left(E_{2}\right)$ is negligible compared to $P\left(E_{1}\right)$ and so the approximation SESR $\approx P\left(E_{1}\right)$ used in Model 2 is validated. Since no results are presented which use Model 3 directly the derivations are omitted here although some results can be found in [4].

\section{Results}

Throughout the rest of the paper, results for ESR have been computed from (1) and (11) and SESR from (7) and (14) for random and burst errors, respectively. BBER has been computed from (3) for random errors and from (3) and (12) for burst errors. Many of the results based on independence assumptions are similar to those derived by Stojkovic and Abe [8]. However, all the work on bursty errors is new. Detailed plots of ESR, BBER, and SESR against the BER are given in [4] and are not repeated here.

\section{BER REQUTREMENTS FOR G.826}

Draft Recommendation G.826 does not define apportionment rules for the originating and terminating country block allowances. The stringency (or otherwise) of G.826 can only be assessed after an apportionment methodology for the block allowances is clarified. In this section, we discuss various scenarios for the apportionment and comment on the equivalent BER requirements for the scenarios in terms of BER masks and simple BER thresholds.

\section{A. Apportionment Scenarios for Terrestrial Radio and Cable Systems}

We consider two types of domestic IEN's according to the length of the longest call distance. A short IEN $(d=2)$ has length $1000 \mathrm{~km}$, and a long IEN $(d=12)$ has length 6000 $\mathrm{km}$. The $d$ parameter gives the number of $500 \mathrm{~km}$ increments in the IEN distance.

Now we choose a part, $Z$, of the originating/terminating country block allowance to be apportioned to the IEN. Therefore, the total IEN allocation is $(Z+d) \%$ of the end-to-end EPO. The rest, $(17.5-Z) \%$ is the apportioned CAN budget. We arbitrarily choose $Z$ from a set of $\{2,15\}$.

For each of the apportionment scenarios, the per hop EPO for a $50 \mathrm{~km}$ hop in the IEN may be found for ESR, BBER and SESR as

$$
\frac{(Z+d)}{100} \times \mathrm{EPO} / 10 \mathrm{~d}
$$

For the CAN, the EPO is simply

$$
\frac{17.5-Z}{100} \times \mathrm{EPO}
$$

It is assumed that the CAN is too short to receive any distance allowance. These EPO may then be converted to equivalent BER using the results of the previous section. 


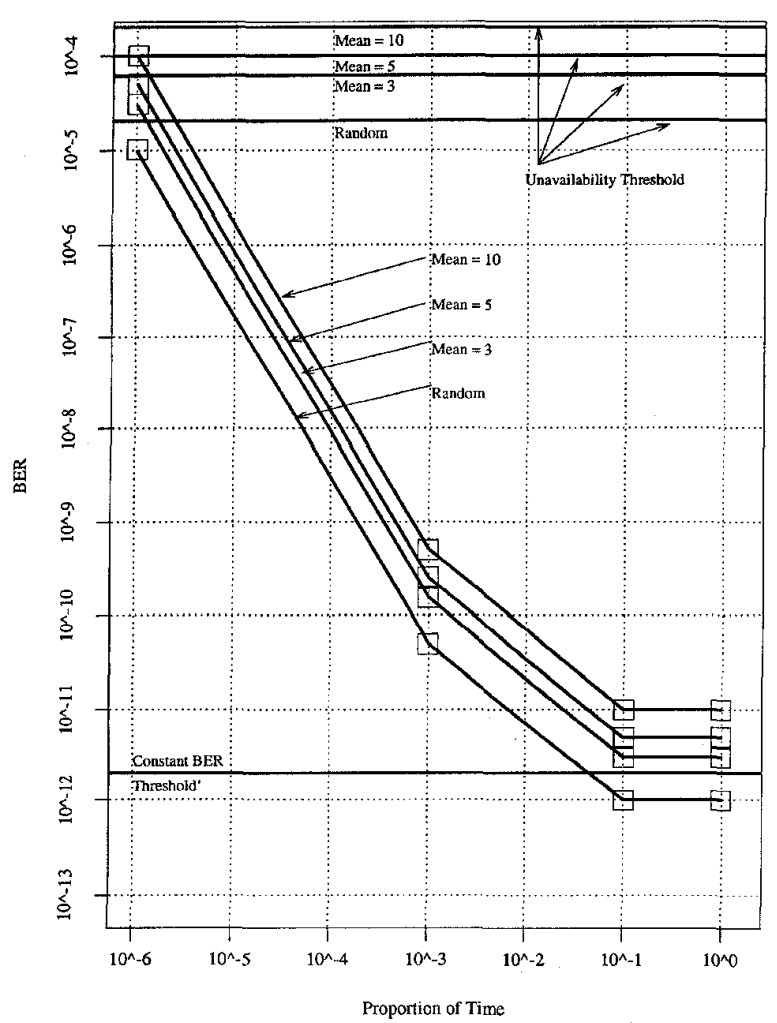

Fig. 3. Mask for a $155 \mathrm{Mb} / \mathrm{s}$ system with 7776 blocks/s $(Z=2, d=2)$.

\section{B. Apportionment for Satellite Systems}

Satellite systems receive a $35 \%$ block allowance and are not given an additional distance-based apportionment.

\section{Summary of Scenarios}

The scenarios considered are summarized below.

S1) A $50 \mathrm{~km}$ hop in a short IEN receiving $2 \%$ block allowance, (see Fig. 3).

$(Z=2, d=2, R=155.52 \mathrm{Mb} / \mathrm{s}, M=7776$ block/s).

S2) A $50 \mathrm{~km}$ hop in a long IEN receiving $2 \%$ block allowance, (see Fig. 4).

$(Z=2, d=12, R=155.52 \mathrm{Mb} / \mathrm{s}, M=7776$ block/s).

S3) CAN receiving $15.5 \%$ block allowance, (see Fig. 5). ( $Z=2, R=2.048 \mathrm{Mb} / \mathrm{s}, M=256 \mathrm{block} / \mathrm{s}$ ).

S4) A $50 \mathrm{~km}$ hop in a short IEN receiving $15 \%$ block allowance, (see Fig. 6).

$(Z=15, d=2, R=155.52 \mathrm{Mb} / \mathrm{s}, M=7776$ block/s).

S5) A $50 \mathrm{~km}$ hop in a long IEN receiving $15 \%$ block allowance, (see Fig. 7).

$(Z=15, d=12, R=155.52 \mathrm{Mb} / \mathrm{s}, M=7776$ block/s).

S6 ) CAN receiving $2.5 \%$ block allowance, (see Fig. 8). ( $Z=15, R=2.048 \mathrm{Mb} / \mathrm{s}, M=256$ block $/ \mathrm{s}$ ).

S7) $\quad 2 \mathrm{Mb} / \mathrm{s}$ satellite link (see Fig. 9). ( $R=2.048 \mathrm{Mb} / \mathrm{s}, M=256 \mathrm{block} / \mathrm{s}$ )

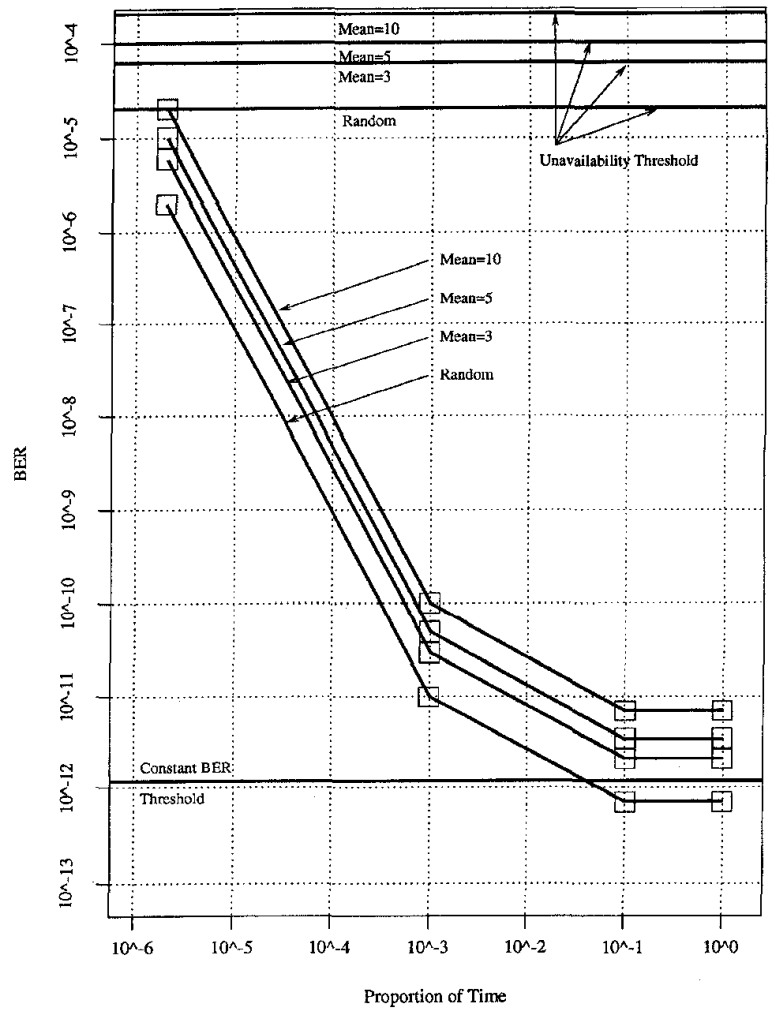

Fig. 4. Mask for a $155 \mathrm{Mb} / \mathrm{s}$ system with 7776 blocks/s $(Z=2, d=12)$.

S8) $\quad 2 \mathrm{Mb} / \mathrm{s}$ satellite link (see Fig. 10). $(R=2.048 \mathrm{Mb} / \mathrm{s}, M=256 \mathrm{block} / \mathrm{s})$.

The EPO's for each scenario are given in Table III.

\section{BER masks}

The equations in Section III and the results given in [4] give simple BER threshold values which are equivalent to the given EPO for fixed BER's. However, over the recommended period of one month total time the BER will not remain constant. Hence, to convert the EPO to equivalent BER values the most useful approach is to derive in some sense a worst-case BER against time distribution(s) which yields the maximum EPO allowed. This distribution(s) (or masks) can be directly compared to individual BER data to check the adherence to G.286. Note that the masks are not unique and an important and useful feature of their use is that the shape can be tailored to meet the needs of individual cases. Their derivation is discussed below.

Consider a log-log plot of the BER distribution over time where the time axis is measured as a proportion. If $n$ points are chosen $\left(t_{1}, p_{1}\right), \cdots,\left(t_{n}, p_{n}\right)$ such that the BER values satisfy $0<p_{1}=p_{2}<p_{3}<\cdots<p_{n}<1$ and the proportion of time values satisfy $0<t_{n}<\cdots<t_{1}=1$ then these points represent a piecewise BER distribution over $\left[t_{n}, 1\right]$. The BER behavior over $\left[0, t_{n}\right]$ is discussed shortly. Since the G.286 EPO are expressed solely in terms of available time it is convenient to identify a threshold BER value, $p_{a}$, which separates available time from unavailable. This threshold $p_{a}$ can be found from SESR plots as the BER which gives SESR 


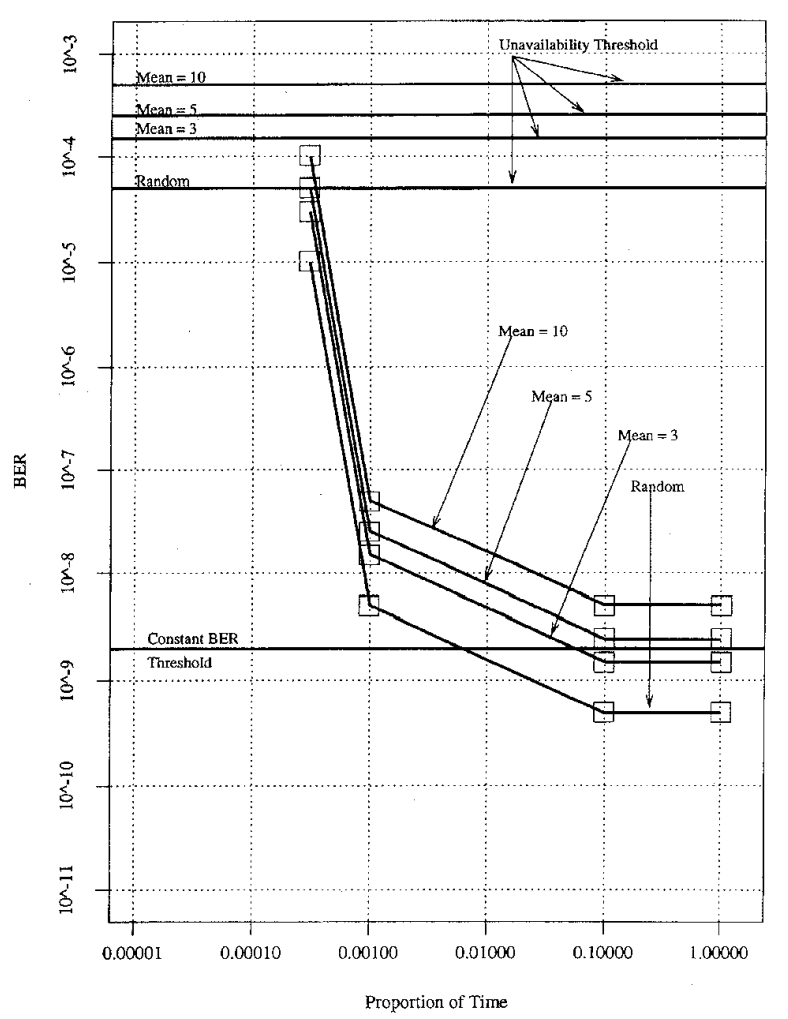

Fig. 5. Mask for a $2 \mathrm{Mb} / \mathrm{s}$ system with 256 blocks/s $(Z=2)$.

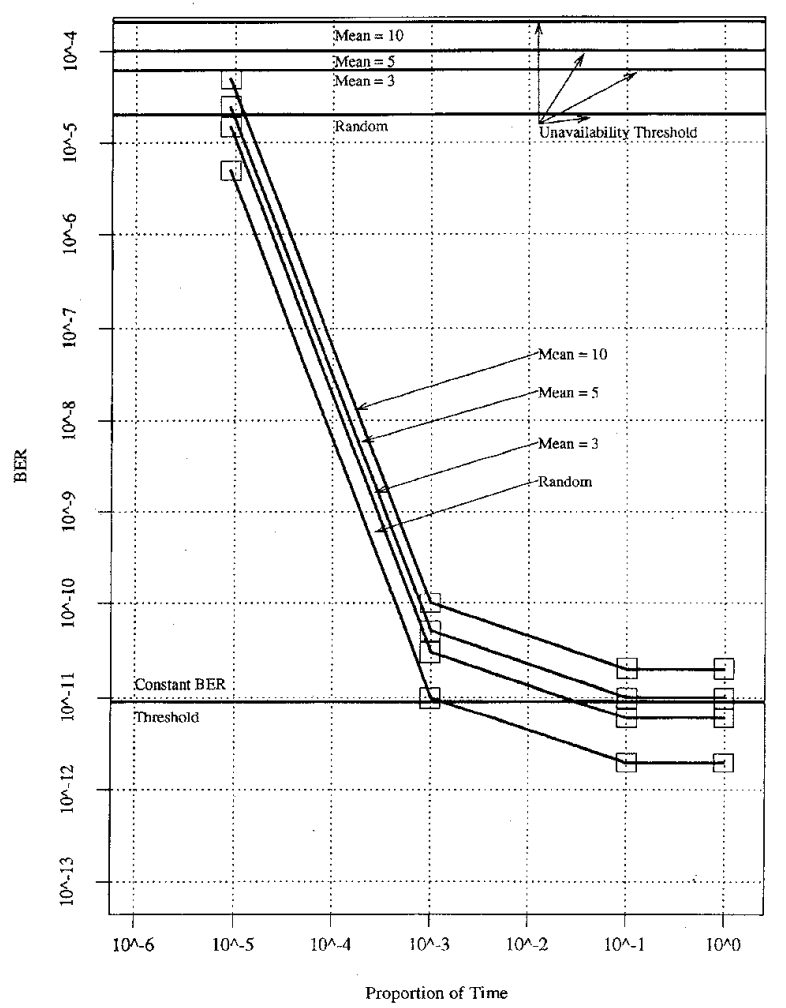

Fig. 6. Mask for a $155 \mathrm{Mb} / \mathrm{s}$ system with 7776 blocks $/ \mathrm{s}(Z=15, d=2)$.

$\approx 1$. The method of choosing $p_{a}$ is discussed in [3], but is described briefly here. Fig. 11 shows a typical SESR against

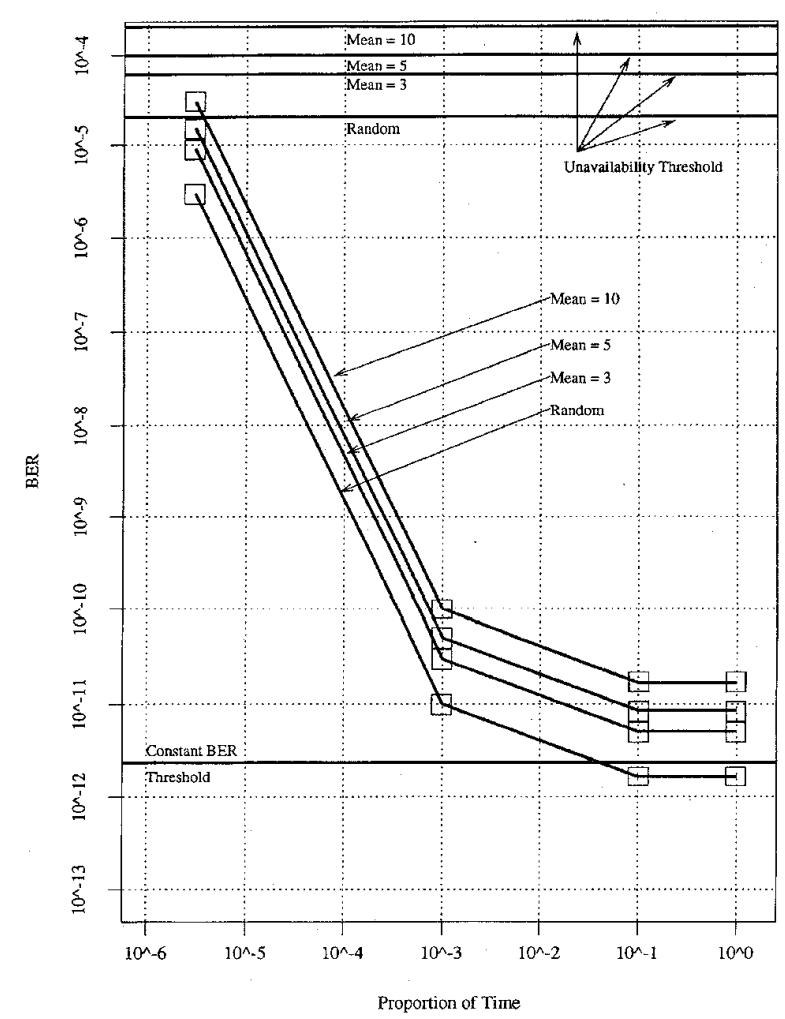

Fig. 7. Mask for a $155 \mathrm{Mb} / \mathrm{s}$ system with 7776 blocks/s $(Z=15, d=12)$.

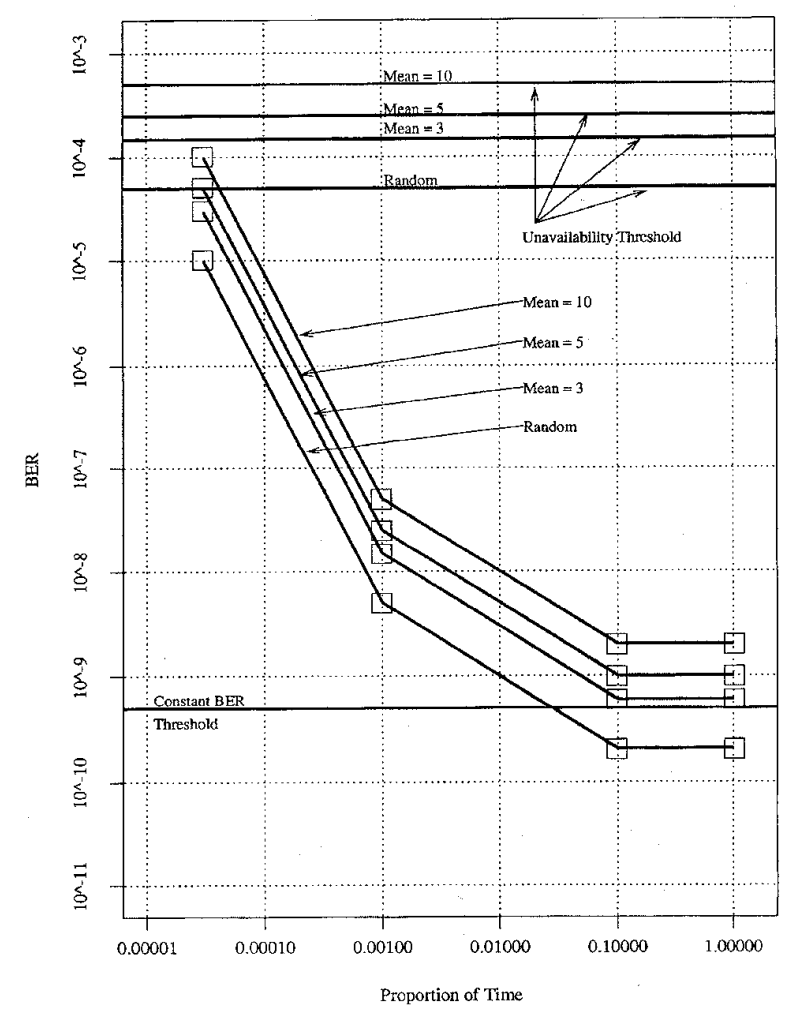

Fig. 8. Mask for a $2 \mathrm{Mb} / \mathrm{s}$ system with 256 blocks/s $(Z=15)$.

BER plot for scenarios S1, S2, S4, and S5. The "brick wall" behavior is clearly shown and a value of $p_{a}=2 \times 10^{-5}$ can 


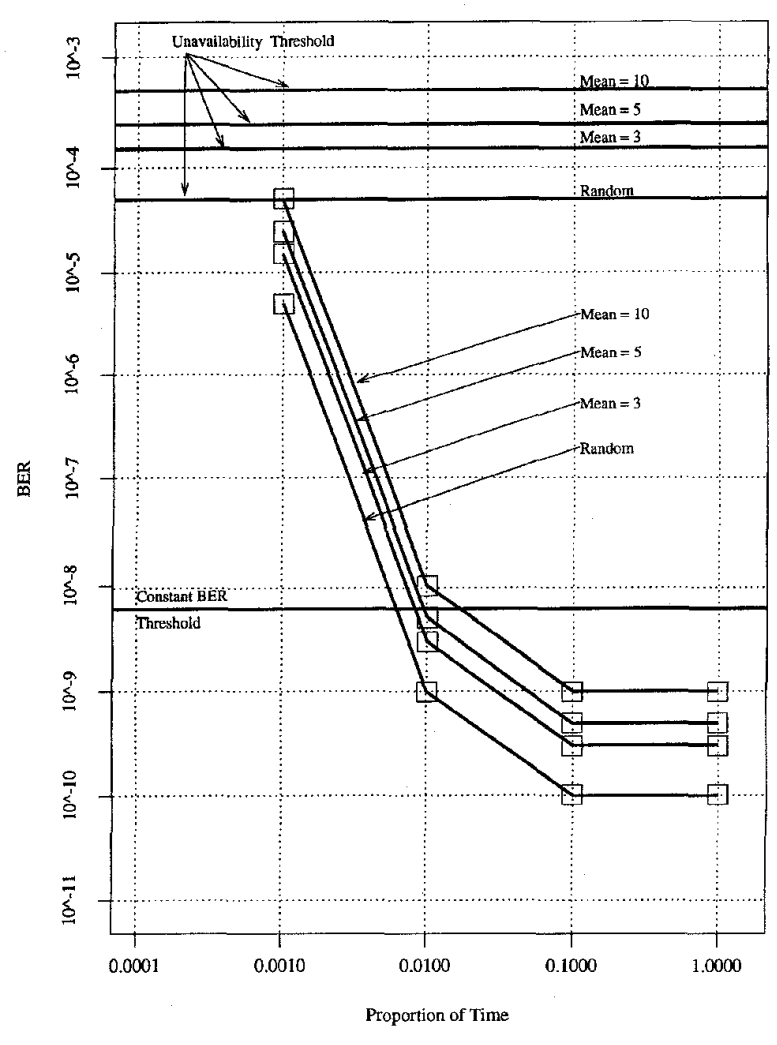

Fig. 9. Mask for a $2 \mathrm{Mb} / \mathrm{s}$ satellite system with 256 blocks/s.

be obtained by extrapolating the nearly vertical section of the random curve to an intercept with SESR $=1$. This is a rather crude approximation since available time will occur at higher error rates than $p_{a}$ and vice versa. However, the extra layer of complexity involved in modeling availability more exactly is probably not worthwhile. The presence of the threshold $p_{a}$ implies the extra condition $p_{n}<p_{a}<1$ on the BER values. Also there is a time $t_{a}$ at which the threshold is reached. The region $\left[0, t_{a}\right]$ corresponds to unavailable time and can be neglected. The BER distribution over $\left[t_{a}, t_{n}\right]$ is given by a straight line on the logarithmic axes joining $\left(t_{a}, p_{a}\right)$ and $\left(t_{n}, p_{n}\right)$. For the case $n=4$ this scenario is illustrated in Fig. 2. In the work that follows $n=4$ is used throughout.

For this distribution to be a mask the total contributions of ESR, SESR, and BBER on the mask line must meet the given EPO values. Ideally, the shape of the mask line is such that all three EPO values are met exactly. However, in practice this is unlikely. To evaluate the contributions on the mask line the following formula is used

$$
\text { Total EPO }=\sum_{i=1}^{r} \operatorname{EPO}\left(p\left(t_{i}\right)\right) \Delta t
$$

where the EPO represents ESR, SESR, or BBER. The time axis is split into $r$ intervals over $\left[t_{a}, 1\right]$ such that $\Delta t=\left(1-t_{a}\right) / r$ and $t_{i}=t_{a}+(i-1) \Delta t$. The BER value on the mask at time $t_{i}$ is denoted $p\left(t_{i}\right)$ and the EPO corresponding to this BER is denoted $\operatorname{EPO}\left(p\left(t_{i}\right)\right)$. These EPO values are found using the results of Section III.

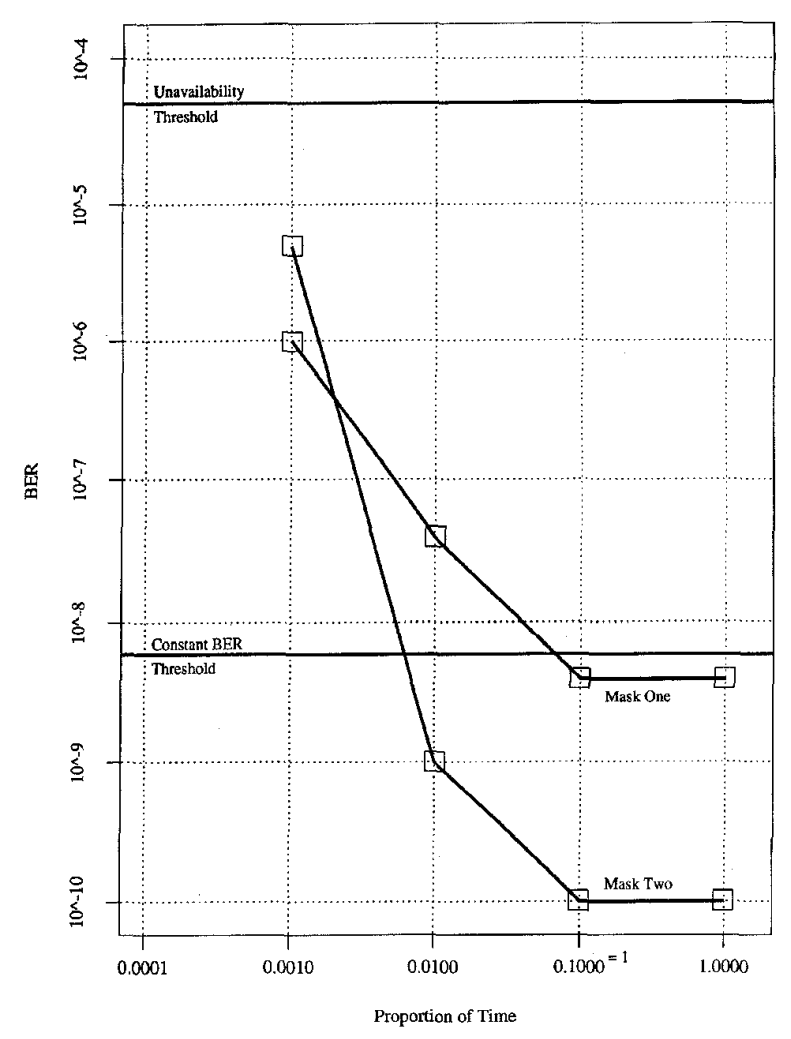

Fig. 10. Masks for a $2 \mathrm{Mb} / \mathrm{s}$ satellite system with 256 blocks/s.

TABLE III

EPO FOR THE VARIOUS SCENARIOS

\begin{tabular}{cccc}
\hline Scenario & ESR EPO & SESR EPO & BBER EPO \\
\hline S1 & $3.2 \times 10^{-4}$ & $4 \times 10^{-6}$ & $4 \times 10^{-7}$ \\
S2 & $1.87 \times 10^{-6}$ & $2.33 \times 10^{-6}$ & $2.33 \times 10^{-7}$ \\
S3 & $6.2 \times 10^{-3}$ & $3.1 \times 10^{-4}$ & $4.65 \times 10^{-5}$ \\
S4 & $1.36 \times 10^{-3}$ & $1.7 \times 10^{-5}$ & $1.7 \times 10^{-6}$ \\
S5 & $3.6 \times 10^{-4}$ & $4.5 \times 10^{-6}$ & $4.5 \times 10^{-7}$ \\
S6 & $10^{-3}$ & $5 \times 10^{-5}$ & $7.5 \times 10^{-6}$ \\
S7 & $1.4 \times 10^{-2}$ & $7 \times 10^{-4}$ & $1.05 \times 10^{-4}$ \\
S8 & $1.4 \times 10^{-2}$ & $7 \times 10^{-4}$ & $1.05 \times 10^{-4}$ \\
\hline
\end{tabular}

In the work that follows we have removed the point $\left(t_{a}, p_{a}\right)$ from the masks to give greater flexibility in the high BER region. This can be done by computing the maximum EPO contributions for any $t_{a} \in\left[0, t_{n}\right]$ and using this worst-case scenario.

The selection of the $n$ mask points is essentially performed by trial and error. A convenient approach is to select $\left(p_{i}, t_{i}\right), i=1, \cdots, n-1$ as required and then iteratively adjust $\left(p_{n}, t_{n}\right)$ to attempt to meet the given EPO. If the EPO cannot be met then the first $n-1$ points must be reset and this process repeated.

\section{E. Masks for the Scenarios}

Using the technique described above, BER masks are computed for the eight scenarios and are given in Figs. 3-10. On each plot separate masks are given for several values of $\mu$, 


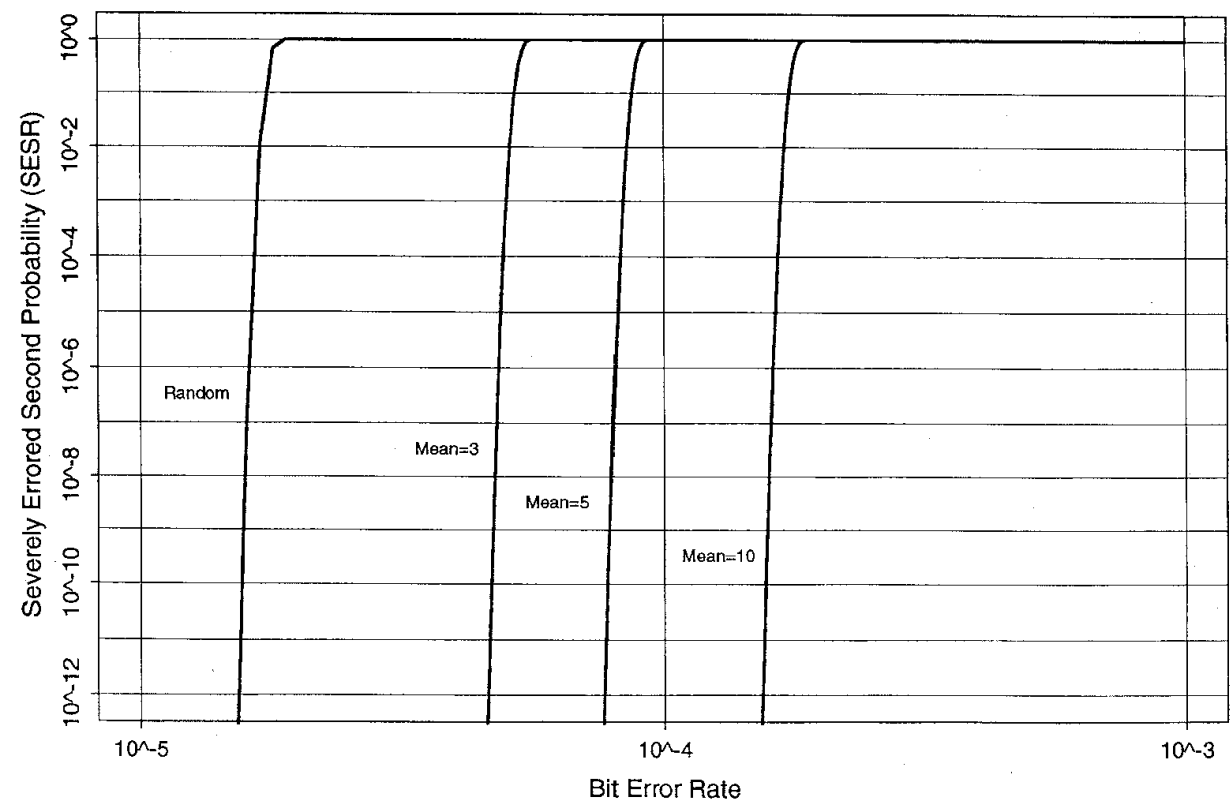

Fig. 11. SESR versus BER for burst errors at $155 \mathrm{Mb} / \mathrm{s}$.

the mean burst length. In addition, the horizontal line shown on each plot is the simple threshold (for the random error case) gained by assuming a constant BER over the whole measurement period. For example, in Fig. 3 the horizontal line at $2 \times 10^{-12}$ indicated that a constant BER of $2 \times 10^{-12}$ will just satisfy G.826.

From Figs. 3-10, it is clear that for all scenarios the use of masks is far more informative than the simple thresholds. In particular, the masks quantify the possible tradeoff of short periods of high BER against lower BER values for the majority of the time. Without this information it would be impossible to tell if the G.826 EPO's were met for any path which occasionally experienced BER values over the threshold. Since the simple thresholds are of the order of $10^{-12}-10^{-9}$ both radio and satellite systems will usually experience periods of operation above this threshold. Hence, for these systems adherence to G.826 cannot be checked by the simple threshold and the use of masks becomes critical.

Another area where masks are essential is in evaluating the relative importance of the three EPO's. For the simple threshold the ESR is always the most critical parameter followed by the BBER and lastly the SESR. By the critical parameter we mean the parameter which first reaches the maximum allowed EPO. Hence, with the simple threshold the constant BER gives you the maximum allowed ESR but the BBER and SESR are below their EPO's. This contradicts experimental findings discussed in ITU Study Group XIII where the BBER was found to be the most important. When more realistic masks are used these findings are verified and the BBER parameter does emerge as the most important EPO, followed by the ESR and lastly the SESR. This result is found whenever the mask allows for high BER values over a period of time which is not negligible. If a flatter mask is used where high BER values cannot occur then the ESR becomes critical again. Hence, as the mask moves from a flat line and becomes increasingly convex so the critical parameter moves from the ESR to BBER. This changeover of the controlling parameter is of course dependent on the block length, bit rate, mean burst length, etc.

The SESR plays the least important role in defining the masks since the SESR is only substantial for a very small proportion of time around the unavailability threshold. The ESR makes contributions over a wide range of BER values and for low error rates would be the most critical since the BBER is small here. Hence, the results from the simple threshold! However, the shape of the more realistic masks gives a small proportion of time to high BER values and here the BBER increases and becomes more significant than the ESR. In addition, the importance of the high BER region supports the need for accurate modeling rather than low BER approximations.

The masks can be used in two ways. Firstly, they provide a direct comparison to measured BER data so that adherence to G.826 can be evaluated. Secondly, they can be used as a design tool. For example, in Fig. 9 the satellite mask assuming random errors can be interpreted as the specifications below.

- BER $\leq 5 \times 10^{-6}$ for at least $99.9 \%$ of total available time.

- BER $\leq 10^{-9}$ for at least $99 \%$ of total available time.

- $\mathrm{BER} \leq 10^{-10}$ for at least $90 \%$ of total available time.

Note that the requirement for the BER to remain below $10^{-10}$ for $90 \%$ of available time necessitates an error floor of around $10^{-11}$, a decade lower than that suggested by the simple BER equivalent!

It can be seen from Figs. 3-9 that the effect of bursty noise is to raise the masks by a nearly linear scaling factor of $\mu$. This is because over a wide range of values the ESR and BBER (the two most critical EPO's) are well approximated as linear functions of the BER. Hence, a scenario with $\mu=10$ will have masks approximately a decade above masks for the random case. 


\section{F. Apportionment}

A comparison of Figs. 3 and 6 and Figs. 4 and 7 shows that the BER masks for the IEN are not sensitive to the large change in apportionment of the block allowance. Comparing Figs. 5 and 8 shows that the mask for the CAN changes more markedly as the allocation to the CAN is decreased from $15.5 \%$ to $2.5 \%$. In particular, the high BER region which is important for the CAN is 10 times wider when the allocation is $15.5 \%$ and there is also a raising of the lower end of the mask.

Thus, it may be appropriate to accord most of the block allowance to the CAN, while the IEN receives the balance of the block allowance and distance-based apportionment.

\section{G. BER Requirements}

The BER values required to satisfy G.826 vary enormously depending on the low performance end of the mask. Fig. 10 illustrates this property for a satellite hop experiencing random errors.

Mask 2 allows BER values $\geq 5 \times 10^{-6}$ to occur for $0.1 \%$ of the time. With this low performance allocation the mask drops to a value of $10^{-10}$ which must be met for $90 \%$ of the time. By reducing the low performance end Mask 1 is produced which allows BER values $\geq 10^{-6}$ for $0.1 \%$ of the time. Mask 1 drops to $4 \times 10^{-9}$ over a decade above Mask 2 ! Hence, the mask shapes are highly sensitive to the left hand points required by a given link.

We can think of the low performance (high BER) end of the mask as controlling the fade margin and the high performance (low BER) end as impacting on the receiver floor. Hence, trading off one against the other to meet G.826 impacts on both the fade margin and the error floor. With this interpretation we can conclude, despite the variation in BER values discussed above, that the following requirements are of the right order of magnitude.

- Satellite systems carrying $2 \mathrm{Mb} / \mathrm{s}$ will require a floor of the order of $10^{-11}-10^{-9}$ depending on the mask chosen and the noise statistics. BER values above $10^{-5}$ are restricted to about $0.1 \%$ of the time (see Figs 9 and 10).

- Depending on the apportionment scheme used the CAN will require a floor of the order of $10^{-11}-10^{-9}$ depending on the mask chosen and the noise statistics. BER values above $10^{-5}$ are restricted to about $0.03 \%$ of the time (see Figs 5 and 8).

- $50 \mathrm{~km}$ hops in IEN will require a floor of $10^{-13}-10^{-12}$ depending on apportionment, the mask chosen and the noise statistics. BER values above $10^{-5}$ are restricted to extremely small time periods of $10^{-4}-10^{-3} \%$ (see Figs 3 , 4,6 , and 7).

The impacts of these requirements are discussed in the conclusions.

\section{Conclusion}

The block-based performance objectives of G.826 can be converted to equivalent BER values for both random and bursty error processes. This is the basis of the creation of BER masks which can be used to check adherence to G.826. An accurate study of the implications of G.826 must be based on BER masks and not simple BER equivalents. In particular we have shown that the use of masks can lead to different conclusions. For example, different error floors and a different understanding of the relative importance of the ESR and BBER objectives are gained by the use of masks. In addition, the masks contain information which is useful in system design and planning since they can be expressed as proportions of time that certain BER thresholds must not be exceeded. This allows a trade-off of normal low BER performance with unusual periods where high BER's are experienced. This is essential in real systems where a constant BER assumption is unrealistic.

Compliance with the new recommendation G.826 will impact on transmission systems in the following ways.

- Assuming $50 \mathrm{~km}$ hop lengths radio relay systems will need to use forward error correction techniques to meet the $10^{-13}-10^{-12}$ error floor requirements, and sophisticated adaptive equalization techniques to restrict error rates above $10^{-5}$ to $10^{-3} \%$ or $10^{-4} \%$ of the time.

- Despite receiving a $35 \%$ block allowance, satellite systems will also need to employ very sophisticated forward error correction techniques to comply with the floor of $10^{-11}-10^{-9}$. Restricting error rates above $10^{-5}$ to less than $0.1 \%$ of the time may also impact on the fade margin since this is usually estimated with respect to the $10^{-3}$ error rate in compliance with G.821.

- Fiber optic systems will comply with all three objectives. However, receiver sensitivities are often quoted at a BER of $10^{-10}$ (ITU 957/958). Compliance with a BER floor requirement of the order of $10^{-12}$ for $50 \mathrm{~km}$ repeater spans is possible, but perhaps at the expense of a system margin which is reserved for system ageing.

The BER requirements of the IEN are not as sensitive to the apportionment of the block allowance as the CAN requirements. Therefore, it may be appropriate to accord most of the block allowance to the CAN, while the IEN receives the balance of the block allowance and the distance-based apportionment. This way, at least the IEN's of originating and terminating countries and transit countries will work to similar performance standards.

\section{APPENDIX A}

DERIVATION OF BBER FOR RANDOM ERRORS

$$
\begin{aligned}
\mathrm{BBER}= & P\left(\mathrm{~EB} \cap \bar{E}_{1} \cap \bar{E}_{2}\right) \\
= & P(\mathrm{~EB} \cap \text { no SDP occurs } \cap \text { number of EB's } \\
& \leq N) \\
= & \sum_{i=0}^{N} P\left(\mathrm{~EB} \cap \text { no SDP occurs } \cap i \mathrm{~EB}^{\prime} \mathrm{s}\right) \\
= & \sum_{i=0}^{N} P\left(\mathrm{~EB} \mid \text { no SDP occurs } \cap i \mathrm{~EB}^{\prime} \mathrm{s}\right) \\
& \cdot P\left(\text { no SDP occurs } \cap i \mathrm{~EB}^{\prime} \mathrm{s}\right) \\
= & \sum_{i=0}^{N} \frac{i}{M} \sum_{j=0}^{i} P\left(\text { no SDP occurs } \cap i-j \mathrm{NSEB}^{\prime} \mathrm{s}\right.
\end{aligned}
$$




$$
\begin{aligned}
& \left.\cap j \mathrm{SEB}^{\prime} \mathrm{s}\right) \\
= & \sum_{i=0}^{N} \frac{i}{M} \sum_{j=0}^{i} N(M-i, i-j, j)\left(1-p_{\mathrm{EB}}\right)^{M-i} \\
& \cdot p_{\mathrm{NSEB}}^{i-j} p_{\mathrm{SEB}}^{j}
\end{aligned}
$$

where $N(M-i, i-j, j)$ is the number of ways you can arrange $M-i$ error free blocks, $i-j$ NSEB's and $j$ SEB's so that no SDP occurs. This number of arrangements can be split into three stages.

Stage 1) Split the $j$ SEB's into $k$ groups of SEB's so that each block has $\leq 3$ SEB's. The number of groups, $k$, can vary from the smallest integer $\geq j / 3$ to $j$.

Stage 2) Arrange the $i-j$ NSEB's and $M-i$ error free blocks in order.

Stage 3) In stage 2, we have created a sequence of $M-j$ blocks. Now choose $k$ from the resulting $M-j+1$ slots for the blocks of SEB's to be located.

For a fixed value of $k$ the number of ways of doing Stage 1 is denoted $N(j, k)$. The numbers of ways of doing Stage 2 and Stage 3 are, respectively,

$$
\left(\begin{array}{c}
M-j \\
i-j
\end{array}\right) \text { and }\left(\begin{array}{c}
M-j+1 \\
k
\end{array}\right)
$$

Hence, we have

$$
N(M-i, i-j, j)=\sum_{k=r}^{j} N(j, k)\left(\begin{array}{c}
M-j \\
i-j
\end{array}\right)\left(\begin{array}{c}
M-j+1 \\
k
\end{array}\right)
$$

where $r$ denotes the smallest integer $\geq j / 3$, and it only remains to prove the recursion for $N(j, k)$.

There is only one way to put $j$ SEB's in $j$ groups and only one way to put $3 j$ SEB's in $j$ groups. Also there is only one way to put two SEB's in one group. Hence, we can define the initial values for a recursion

$$
N(j, j)=N(3 j, j)=N(2,1)=1 \quad \text { for } \quad j=1, \cdots, N \text {. }
$$

Values "outside" of these initial values are zero, hence

$$
N(j, k)=0 \text { for } j>3 k \text { or } j<k \text {. }
$$

The recursion stems from the following breakdown:

$j$ blocks in $k$ groups $\equiv j-i$ blocks in the first $k-1$ groups and $i$ blocks in the last group

$$
N(j, k)=\sum_{i} N(j-i, k-1)
$$

The summation can be over $i=1,2,3$ but if $j-i<k-1$ or $j-i>3(k-1)$ the terms are zero, hence, the summation in (2).
APPENDIX B

DERIVATION OF SESR FOR RANDOM ERRORS

$$
\begin{aligned}
\mathrm{SESR}= & 1-P\left(\bar{E}_{1} \cap \bar{E}_{2}\right) \\
= & 1-P(\text { no SDP occurs } \cap \text { number of EB's } \leq N) \\
= & 1-\sum_{i=0}^{N} P\left(\text { no SDP occurs } \cap i \mathrm{~EB}^{\prime} \mathrm{s}\right) \\
= & 1-\sum_{i=0}^{N} \sum_{j=0}^{i} \sum_{k=r}^{j} N(j, k)\left(\begin{array}{c}
M-j+1 \\
k
\end{array}\right)\left(\begin{array}{c}
M-j \\
i-j
\end{array}\right) \\
& \cdot p_{\mathrm{SEB}}^{j}\left(1-p_{\mathrm{EB}}\right)^{M-i} p_{\mathrm{NSEB}}^{i-j} .
\end{aligned}
$$

directly from Appendix A.

\section{REFERENCES}

[1] CCITT Study Group XVIII, Recommendation G.826: Error Performance Parameters and Objectives for International Constant Bit Rate Digital Paths At or Above the Primary Rate, Geneva, Switzerland, June 1993.

[2] CCITT Study Group XVIII. Recommendation G.82l: Error Performance of an International Digital Connection Forming Part of an ISDN, Geneva, Switzerland, June 1980.

[3] ITU-R Working Party 4B, Recommendation S.1062 : Allowable Error Performance for a Hypothetical Reference Digital Path Operating at or above the Primary Rate, Feb. 1995.

[4] M. Shafi and P. Smith, "The Impact of G.826," IEEE Commun. Mag., vol. 31, pp. 56-62, Sept. 1993.

[5] J. R. Gray, Probability. Edinburgh, U.K.: Oliver and Boyd Ltd, 1967.

[6] G. G. Pullum, "Modeling burst errors in digital transmission," in Mathematical Topics in Telecommunications, Problems of Randomness in Communications Engineering, vol 2, K. W. Cattermole and J. J. O'Reilly, Eds. London, U.K.: Pentech Press, 1984.

[7] J. J. Hunter, Mathematical Techniques of Applied Probability. New York: Academic, 1983.

[8] M. Stojkovic and M. Abe, Draft New Recommendation ZZZ: Allowable Error Performance for a Hypothetical Reference Digital Path Operating at or above the Primary Rate, Document 4B/TEMP/35-E, CCITT Study Group XVIII, May 1992.

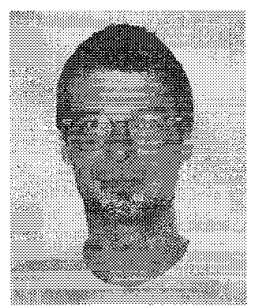

Peter J. Smith (M'93) received the B.Sc. degree in mathematics and the Ph.D. degree in statistics from the University of London, London, U.K., in 1983 and 1988 , respectively.

From 1983 to 1986, he was with the Telecommunications Laboratories at GEC Hirst Research Centre. Since 1988, he has been a Lecturer in statistics at Victoria University, Wellington, New Zealand. His research interests include the statistical aspects of design and analysis for communication systems, especially coherent optical systems, antenna arrays, and mobile radio.

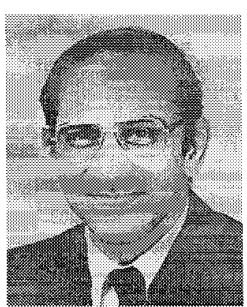

Mansoor Shafi (S'69-A'70-M'82-SM'87-F'93) received the B.S. degree from Engineering University, Lahore, Pakistan, and the Ph.D. degree from the University of Auckland, Auckland, New Zealand, in 1970 and 1979, respectively, both in electrical engineering.

Since 1979, he has held different positions with the New Zealand Post Office and then with Telecom New Zealand. His research interests are in fixed and mobile transmission systems.

Dr. Shafi was awarded the 1992 IEEE Communications Society Public Service Award in Communications. 